\title{
MR Perfusion to Determine the Status of Collaterals in Patients with Acute Ischemic Stroke: A Look Beyond Time Maps
}

\author{
(D). Nael, (D) A. Doshi, (D). De Leacy, (D). Puig, (D). Castellanos, (D). Bederson, (D)T.P. Naidich, (D) Mocco, and (D). Wintermark
}

\begin{abstract}
BACKGROUND AND PURPOSE: Patients with acute stroke with robust collateral flow have better clinical outcomes and may benefit from endovascular treatment throughout an extended time window. Using a multiparametric approach, we aimed to identify MR perfusion parameters that can represent the extent of collaterals, approximating DSA.
\end{abstract}

MATERIALS AND METHODS: Patients with anterior circulation proximal arterial occlusion who had baseline MR perfusion and DSA were evaluated. The volume of arterial tissue delay (ATD) at thresholds of $2-6$ seconds (ATD ${ }^{2-6}$ seconds) and $>6$ seconds (ATD ${ }^{>6}$ seconds) in addition to corresponding values of normalized CBV and CBF was calculated using VOI analysis. The association of MR perfusion parameters and the status of collaterals on DSA were assessed by multivariate analyses. Receiver operating characteristic analysis was performed.

RESULTS: Of 108 patients reviewed, 39 met our inclusion criteria. On DSA, 22/39 (56\%) patients had good collaterals. Patients with good collaterals had significantly smaller baseline and final infarct volumes, smaller volumes of severe hypoperfusion (ATD $>6$ seconds), larger volumes of moderate hypoperfusion ( $A T D^{2-6}$ seconds), and higher relative CBF and relative CBV values than patients with insufficient collaterals. Combining the 2 parameters into a Perfusion Collateral Index (volume of ATD ${ }^{2-6}$ seconds $\times$ relative CBV ${ }^{2-6}$ seconds) yielded the highest accuracy for predicting collateral status: At a threshold of 61.7, this index identified 15/17 (88\%) patients with insufficient collaterals and $22 / 22(100 \%)$ patients with good collaterals, for an overall accuracy of $94.1 \%$.

CONCLUSIONS: The Perfusion Collateral Index can predict the baseline collateral status with $94 \%$ diagnostic accuracy compared with DSA.

ABBREVIATIONS: ASITN = American Society of Interventional and Therapeutic Neuroradiology; ATD = arterial tissue delay; PCI = Perfusion Collateral Index; $\mathrm{rCBF}=$ relative cerebral blood flow; $\mathrm{rCBV}=$ relative cerebral blood volume; $\mathrm{ROC}=$ receiver operating characteristic; Tmax $=$ time-to-maximum

n patients with acute ischemic stroke, the status of collateral blood supply can be an independent predictor of reperfusion success and can impact the clinical outcome. ${ }^{1,2}$ In addition, promising data suggest that good collateral status can prolong the

Received June 11, 2017; accepted after revision September 14.

From the Departments of Radiology (K.N., A.D., T.P.N.) and Neurosurgery (R.D.L., J.B., JM.), Icahn School of Medicine at Mount Sinai, New York, New York; Department of Radiology (I.P.), Girona Biomedical Research Institute, Diagnostic Imaging Institute, Hospital Universitari Dr Josep Trueta, Girona, Spain; Department of Neurology (M.C.), A Coruña University Hospital, A Coruña Biomedical Research Institute, A Coruña, Spain; and Department of Radiology (M.W.), Neuroradiology Section, Stanford University, Palo Alto, California.

This article has not been submitted or published elsewhere in whole or in part, except as an abstract (oral presentation, No. A61) at: 2016 International Stroke Conference, February 16-19, 2016; Los Angeles, California; and as an oral presentation at: Radiological Society of North America 102nd Scientific Assembly and Annual Meeting, November 27-December 2, 2016; Chicago, Illinois.

Please address correspondence to Kambiz Nael, MD, Department of Radiology, Icahn School of Medicine at Mount Sinai, One Gustave L. Levy Place, Box 1234 , New York, NY 10029-6574; e-mail: Kambiznael@gmail.com; @kambiznael

- Indicates open access to non-subscribers at www.ajnr.org

http://dx.doi.org/10.3174/ajnr.A5454 time that tissue-at-risk remains salvageable, ${ }^{3-5}$ which, in turn, may allow extension of the therapeutic window. Therefore, noninvasive evaluation of the collateral blood supply may be helpful in identifying patients who may benefit from endovascular thrombectomy.

Noninvasive imaging of collateral status can be assessed by direct visualization of the collateral vessels with $\mathrm{CTA}^{6,7}$ or $\mathrm{MRA}^{8,9}$ or by the assessment of the efficiency of collateral perfusion with CT perfusion ${ }^{10,11}$ or MR perfusion. ${ }^{12-14}$ These techniques are complementary: CTA and MRA provide anatomic information about collateral vessels, while perfusion techniques provide functional and circulatory information on leptomeningeal and secondary collateral pathways. ${ }^{15}$ Although various perfusion parameters have been used to measure collateral status, specific perfusion criteria to assess collateral status have yet to be defined. Most research has focused on time-based perfusion maps, assuming that patients with good collaterals have less severe delays ${ }^{12}$ and larger perfusion delay volumes. ${ }^{13,16}$ Some investigators have also looked into using CBV and CBF from raw perfusion data to assess collateral status. $^{12,17}$ 
In this study, we hypothesized that MR perfusion time maps, which show only delayed perfusion, are insufficient to predict the status of collaterals if used alone. By applying a multiparametric approach, we aimed to identify perfusion parameters that can represent the extent of collaterals, approximating DSA.

\section{MATERIALS AND METHODS}

\section{Patients}

Patients with anterior circulation acute ischemic stroke who presented within 9 hours of symptom onset and who were considered for revascularization treatment were retrospectively reviewed under an approved institutional review board protocol. Inclusion criteria were the following: 1) large unilateral proximal arterial occlusion (internal carotid or proximal middle cerebral artery), 2) baseline MR imaging including DSC perfusion and DSA, and 3) follow-up imaging to determine the final infarction volume. Patients were excluded if DSC perfusion was nondiagnostic or DSA studies did not allow adequate evaluation of collaterals.

We recorded clinical data, including patient age, sex, baseline NIHSS scores, time from stroke onset, time from onset to groin puncture, and treatment type, including intravenous tissue plasminogen activator (IV tPA) and/or mechanical thrombectomy when available.

\section{Image Analysis}

DWI was acquired using a single-shot spin-echo EPI sequence with following parameters: TR/TE, 4900/98 ms; flip angle, $90^{\circ}$; FOV, $22 \times 22 \mathrm{~cm}$; matrix, $128 \mathrm{~mm}$; sections, $30 \times 5 \mathrm{~mm}$. Diffusion gradients were applied along 6 noncollinear directions with b-values of 0 and $1000 \mathrm{~s} / \mathrm{mm}^{2}$, resulting in a 51 -second acquisition time. DSC perfusion was performed with a single-shot gradient-echo EPI sequence with the following parameters: TR/TE, 1450/22 ms; flip angle, 90 ${ }^{\circ}$; FOV, $22 \times 22 \mathrm{~cm}$; matrix, $128 \mathrm{~mm}$; sections, $30 \times 4 \mathrm{~mm}$. Sixty dynamic frames were obtained during a 90-second acquisition time. A generalized autocalibrating partially parallel acquisition technique with an acceleration factor of 2 was used for both DWI and DSC.

DSC perfusion was processed with US Food and Drug Administration-approved software (Olea Sphere; Olea Medical, La Ciotat, France) by applying a Bayesian probabilistic method. ${ }^{18}$ Bayesian is a delay-insensitive probabilistic method in which the Bayes rule is applied to combine experimental perfusion data and a priori information about the parameters, to compute a posteriori probability distribution functions for every parameter of interest. From these distributions, parameter estimates and errors on those estimates can be derived (eg, the mean and SD of the posteriori distributions). Arterial tissue delay (ATD), defined as time-tomaximum of the Bayesian-estimated tissue residue function, $\mathrm{CBF}$, and $\mathrm{CBV}$, was generated. The software used a 6- $d f$ transformation and a mutual information cost function to coregister DWI and all perfusion maps for each patient. DSC maps were normalized to an ROI placed in normal-appearing white matter in the centrum semiovale, contralateral to the infarct side, to calculate the ratios for $\mathrm{CBV}$ and $\mathrm{CBF}$ values.

Because ATD is essentially a Bayesian-estimated time-tomaximum (Tmax), previously used time-delay thresholds of 2 and 6 seconds ${ }^{12,13,19}$ were used to differentiate brain tissue perfusion properties. ATD $>2$ seconds defined any visually perceptible delayed perfusion, and ATD maps with thresholds of 2 and 6 seconds were generated. We applied the following designations: 1) Tissue with ATD $>6$ seconds was defined as having severe delayed perfusion; 2) tissue with 2 seconds $<$ ATD $\leq 6$ seconds was defined as having moderately delayed perfusion.

Subsequently, a VOI from the above-described ATD maps was calculated using a voxel-based signal intensity threshold method subsuming the entire region of hypoperfusion within the defined threshold. The volume of each VOI and its corresponding relative cerebral blood flow (rCBF) and relative cerebral blood volume (rCBV) were calculated for each patient. Assuming that most blood volume in the moderate hypoperfused tissue is likely via collaterals, we calculated a new perfusion index as the Perfusion Collateral Index (PCI), defined as the volume of moderately hypoperfused tissue $\left(\mathrm{ATD}^{2-6 \text { seconds }}\right)$ multiplied by its corresponding mean $\mathrm{rCBV}$ $\left(\mathrm{PCI}=\right.$ Volume of $\left.\mathrm{ATD}^{2-6 \text { seconds }} \times \mathrm{rCBV}^{2-6 \text { seconds }}\right)$.

We defined the baseline infarct volume as the volume of tissue with an apparent diffusion coefficient $<600 \times 10^{-6} \mathrm{~mm}^{2} / \mathrm{s}$ on the baseline MR diffusion images. ${ }^{20}$ Final infarct volume was calculated from the follow-up imaging (obtained within 7 days from the initial imaging) using the volume of FLAIR-hyperintense tissue or CT-hypodense tissue.

\section{DSA Analysis}

Angiographic collateral grading was performed with the American Society of Interventional and Therapeutic Neuroradiology (ASITN)/Society of Interventional Radiology Collateral Flow Grading System on baseline angiography (ASITN grades $0-4)^{21}$ : grade 0 : no collaterals visible; grade 1: slow collaterals to the periphery of the ischemic site with persistence of some of the defect; grade 2: rapid collaterals to the periphery of the ischemic site with persistence of some of the defect; grade 3: collaterals with slowbut-complete angiographic blood flow of the ischemic bed by the late venous phase; and grade 4: complete and rapid collateral blood flow to the vascular bed in the entire ischemic territory by retrograde perfusion.

Patients were dichotomized to those with good collaterals (ASITN grades 3 and 4) and insufficient collaterals (ASITN grades 0,1 , and 2). Primary revascularization following mechanical thrombectomy was assessed with the TICI scale. ${ }^{22}$ Data were dichotomized with TICI $\geq 2 \mathrm{~b}$ as an indication of successful revascularization.

\section{Statistical Analysis}

Baseline characteristics and neuroimaging variables were compared between subjects with insufficient-versus-good collaterals using the Fisher exact and Wilcoxon rank sum tests as appropriate. For multivariate analysis, a Classification Tree (binary recursive partition) model was used to simultaneously evaluate all ( $n=$ 12 ) included imaging (baseline infarction volume, final infarction volume, $\mathrm{ATD}^{>6 \text { seconds }}$, $\mathrm{ATD}^{2-6}$ seconds, $\mathrm{rCBF}, \mathrm{rCBV}$, and $\mathrm{PCI}$ ) and clinical (age, sex, baseline NIHSS score, IV tPA administration, time-to-groin puncture from last known well) parameters of insufficient-versus-good collaterals. 
Table 1: Baseline and clinical data in patients with insufficient-versus-good collateral flow

\begin{tabular}{|c|c|c|c|c|}
\hline Variable & $\begin{array}{l}\text { Overall } \\
(n=39)\end{array}$ & $\begin{array}{c}\text { Good } \\
\text { Collaterals } \\
(n=22)\end{array}$ & $\begin{array}{c}\text { Insufficient } \\
\text { Collaterals } \\
\text { ( } n=17)\end{array}$ & $\begin{array}{c}P \\
\text { Value }\end{array}$ \\
\hline Age (mean) (SD) (yr) & $63.3(13.3)$ & $62.8(15.3)$ & $63.9(10.2)$ & .85 \\
\hline $\operatorname{Sex}(M / F)$ & $(22: 17)^{\prime}$ & (11:11) & $(11: 6)$ & .52 \\
\hline Baseline NIHSS score (median) (IQR) & $17,14-19$ & $16,13-18$ & $18,15-20$ & .19 \\
\hline IV tPA (No.) (\%) & $18(46 \%)$ & $10(45 \%)$ & $8(47 \%)$ & .45 \\
\hline $\mathrm{Hr}$ to groin puncture from last known well (median) (IQR) & $6.10,5.54-7.14$ & $5.98,5.67-7.54$ & $6.08,5.27-7.10$ & .93 \\
\hline Hr from MRI to groin puncture (median) (IQR) ${ }^{a}$ & $1.40,0.80-2.20$ & $1.10,0.70-1.75$ & $1.5,0.90-2.14$ & .58 \\
\hline
\end{tabular}

Note:-IQR indicates interquartile range.

${ }^{\text {a }}$ Subset analysis (only available for 20 patients, 13 patients with good collaterals and 7 patients with insufficient collaterals).

Table 2: Imaging data in patients with insufficient-versus-good collateral flow

\begin{tabular}{|c|c|c|c|}
\hline Variable & $\begin{array}{c}\text { Good } \\
\text { Collaterals } \\
(n=22)\end{array}$ & $\begin{array}{c}\text { Insufficient } \\
\text { Collaterals } \\
\text { ( } n=17)\end{array}$ & $\begin{array}{c}P \\
\text { Value }\end{array}$ \\
\hline Baseline infarct volume (mean) (SD) & $29.7(18.7)$ & $48.3(30.0)$ & $.021^{\mathrm{a}}$ \\
\hline Final infarct volume (mean) (SD) & $51.1(32.4)$ & $81.0(44.7)$ & $.030^{\mathrm{a}}$ \\
\hline $\mathrm{ATD}^{>6 \mathrm{~s}}(\mathrm{vol})$ (mean) (SD) & $24.7(17.4)$ & $42.4(18.2)$ & $.004^{\mathrm{a}}$ \\
\hline ATD $^{2-6 \text { s }}$ (vol) (mean) (SD) & $64.6(25.0)$ & $28.2(17.1)$ & $<.001^{\mathrm{a}}$ \\
\hline $\mathrm{rCBF}^{2-6 \mathrm{~s}}$ (mean) (SD) & $1.16(0.18)$ & $0.98(0.25)$ & $.026^{\mathrm{a}}$ \\
\hline $\mathrm{rCBV}^{2-6} \mathrm{~s}$ (mean) (SD) & $1.92(0.32)$ & $1.17(0.38)$ & $<.001^{\mathrm{a}}$ \\
\hline $\mathrm{PCl}$ (mean) (SD) & $120.70(47.6)$ & $36.0(25.8)$ & $<.001^{\mathrm{a}}$ \\
\hline $\begin{array}{l}\text { Primary revascularization } \\
\text { (DSA-TICI) (No.) (\%) }\end{array}$ & $12(54.5)$ & $5(29.4)$ & .190 \\
\hline
\end{tabular}

Note:-vol indicates volume.

a Significant.

${ }^{\mathrm{b}}$ Primary revascularization based on the outcome of thrombectomy, dichotomized to 0 and 1 using $\mathrm{TICl} \geq 2 \mathrm{~b}$.
$(8 / 17,47 \%)$ (Table 1$)$. The time gap between MR imaging and DSA was not significantly different between patients with good collaterals (mean, $100 \pm 45$ minutes) versus insufficient collaterals (mean, $128 \pm 39$ minutes). Endovascular treatment was performed with a Merci retriever (Concentric Medical, Mountain View, California) catheter $(n=12)$ or the Penumbra (Penumbra, Alameda, California) suction thrombectomy catheter $(n=16)$ and stent retrieval device $(n=11)$.
Accuracy statistics were computed with a nonparametric receiver operating characteristic (ROC) analysis. Thresholds for separating insufficient from good collaterals were chosen to maximize the unweighted overall accuracy, defined as the weighted average of the percentage of insufficient and good collateral vessels. Sensitivity, specificity, overall accuracy defined as (sensitivity + specificity) / 2 , and the area under the ROC curve were reported. The significance level was set at $P=.05$ in our statistical analysis.

\section{RESULTS}

Among 108 charts reviewed, 39 patients met our inclusion criteria. In 61 patients, baseline DSA was inadequate to provide a score of collateral vessels (lack of an adequate number of phases and injections). Eight patients were excluded due to inadequate and nondiagnostic MR perfusion data. Of those patients included in the analysis, 22 were men, 17 were women (mean age, 63.3 years; range, 37-85 years). The median and interquartile range of the NIHSS scores were 17 and 14-19. Five patients had distal internal carotid occlusion, and 34 patients had proximal MCA occlusion. Twenty-two (56\%) patients were classified as having good collaterals defined by ASITN $\geq 3$ on baseline conventional angiography.

\section{Clinical Data}

Demographic data and basic clinical information for patients with insufficient-versus-good collaterals are provided in Table 1. Among the 39 patients included, 18 (46\%) received IV tPA before undergoing conventional angiography. Administration of IV tPA was not significantly different $(P=.45)$ between patients with good $(10 / 22,45 \%)$ versus insufficient collaterals

\section{Imaging Data}

Univariate analysis showed significantly smaller baseline infarction volume, final infarction volume, and volume of severe (ATD $^{>6}$ seconds $)$ delayed perfusion in patients with good collaterals (Table 2). On the other hand, rCBF, rCBV, PCI, and the volume of moderate $\left(\mathrm{ATD}^{2-6}\right.$ seconds $)$ delayed perfusion were significantly higher in patients with good collaterals (Table 2). The rate of primary postprocedural revascularization did not differ significantly between the 2 groups (Table 2). Figs 1 and 2 show examples of our image analysis in 2 patients with right proximal MCA occlusion; one had good and the other had insufficient collaterals, respectively.

The Classification Tree (binary recursive partition) model in the simultaneous evaluation of all 12 included predictive parameters of insufficient-versus-good collaterals showed that the 2 best single parameters with the highest predictive ability were $\mathrm{rCBV}^{2-6}$ seconds and volume of moderate (ATD ${ }^{2-6 \text { seconds }}$ ) hypoperfusion, with an overall diagnostic accuracy of $85 \%$ and $82.1 \%$, respectively. Both $\mathrm{rCBV}^{2-6}$ seconds (at a threshold of $>1.6$ ) and volume of ATD $^{2-6}$ seconds (at a threshold of $>38.28 \mathrm{~mL}$ ) could identify 18/22 $(81.8 \%)$ patients with good collaterals when used alone. A combination of the 2 parameters used in our newly defined Perfusion Collateral Index (Volume of $\mathrm{ATD}^{2-6 \text { seconds }} \times \mathrm{rCBV}^{2-6 \text { seconds }}$ ) resulted in improved predictive accuracy over each measure alone to correctly identify all 22 patients (100\%) with good collaterals. Model accuracy statistics, including sensitivity, specificity, overall accuracy, and area under the ROC curve in addition to the optimal threshold for the variables that reached statistical significance, are reported in Table 3. In further analysis of our Classification Tree model, a combination of PCI and baseline infarction volume showed further improvement in predictive accuracy, providing the best tree model with a nominal (not validated) 100\% accuracy and ROC area $=1.0$ (Fig 3). 

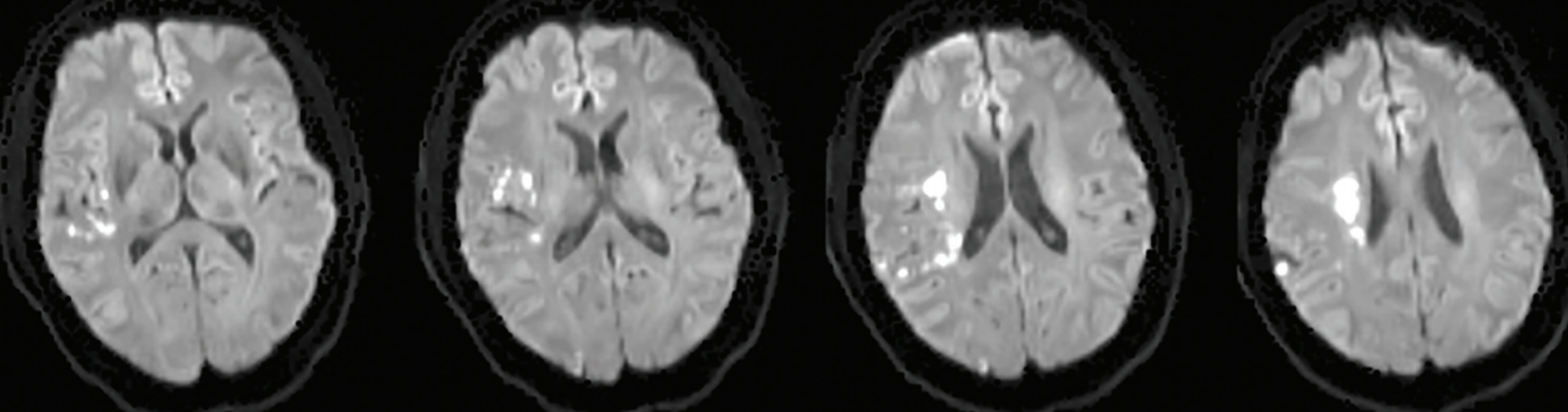

DWI

\section{ATD $>6 s e c$}

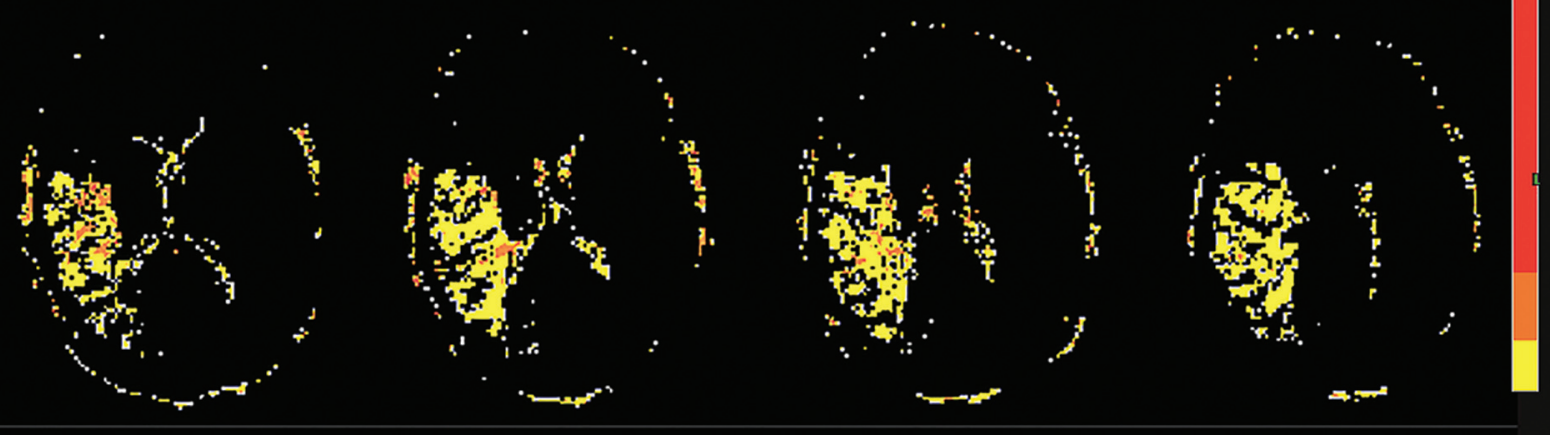

\section{ATD 2-6sec}

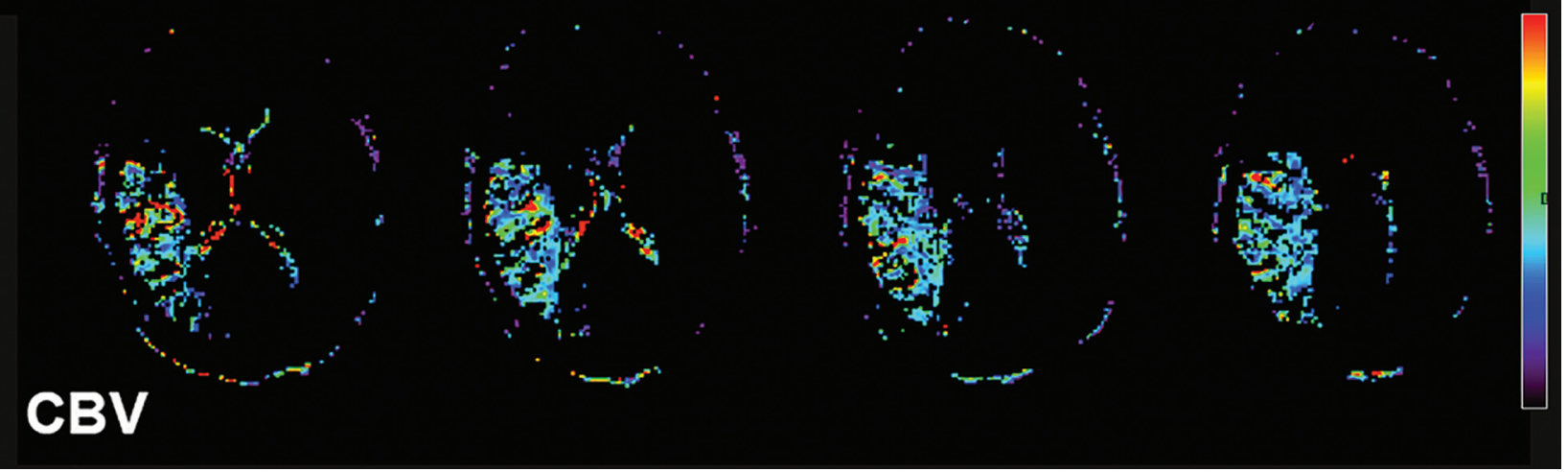

FIG 1. A 61-year-old woman with left hemiparesis who had right MCA (M1) occlusion (not shown) but sufficient collaterals on baseline conventional angiography. DWI shows right MCA territorial infarction. Processed perfusion maps show $3.5 \mathrm{~mL}$ of severe (ATD $>6$ seconds) hypoperfusion, $42 \mathrm{~mL}$ of moderate (ATD ${ }^{2-6}$ seconds) hypoperfusion, and a mean rCBV ${ }^{2-6}$ seconds of 1.7 within the hypoperfused area. The Perfusion Collateral Index is $42 \times 1.7=71.4$.

\section{DISCUSSION}

In this study, we showed that multiparametric MR perfusion enables accurate assessment of collateral status in patients with anterior circulation proximal arterial occlusion who may be candi- dates for endovascular revascularization. By incorporating rCBV in addition to commonly used perfusion time maps, we defined the Perfusion Collateral Index as a new perfusion parameter superior to other baseline imaging variables to predict the status of 


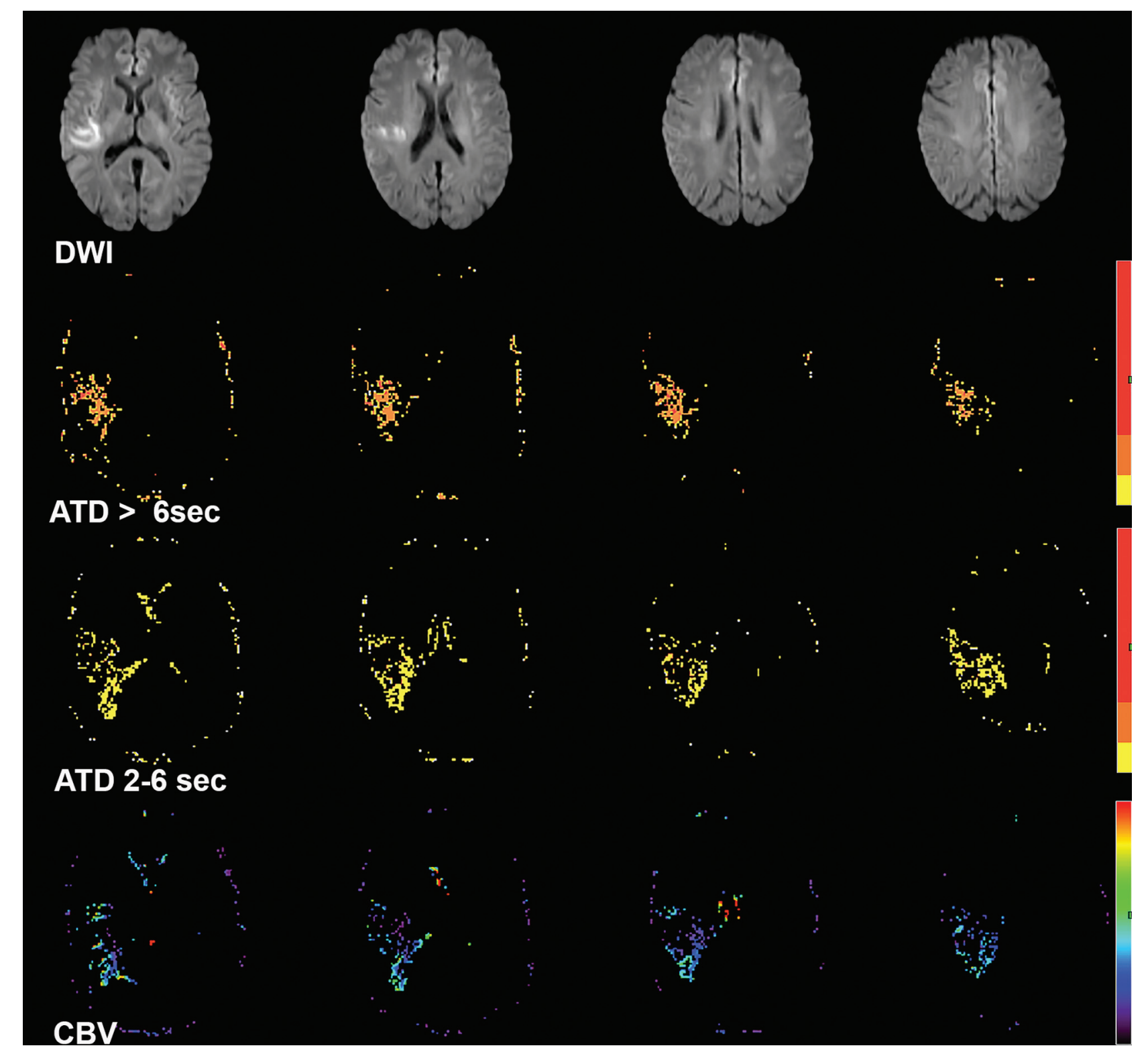

FIG 2. A 70-year-old woman with left paresis who had right MCA (M1) occlusion (not shown) and insufficient collaterals on baseline conventional angiography. DWI showed right MCA territorial infarction. Processed perfusion maps show $17 \mathrm{~mL}$ of severe (ATD $>6$ seconds) hypoperfusion, $20 \mathrm{~mL}$ of moderate (ATD ${ }^{2-6}$ seconds) hypoperfusion, and a mean $\mathrm{rCBV}^{2-6}$ seconds of 0.9 within the hypoperfused area. The perfusion collateral index is $20 \times 0.9=18$.

Table 3: Optimal threshold, sensitivity, specificity, overall accuracy, and ROC area imaging variables that were significant with univariate analysis

\begin{tabular}{|c|c|c|c|c|c|}
\hline Variable & Threshold & Specificity & Sensitivity & $\begin{array}{c}\text { Overall } \\
\text { Accuracy }\end{array}$ & $\begin{array}{l}\text { ROC } \\
\text { Area }\end{array}$ \\
\hline Base infarct volume (mL) & 24.1 & $76.5 \%$ & $63.6 \%$ & $70.1 \%$ & 0.717 \\
\hline Final infarct volume (mL) & 45 & $82.4 \%$ & $59.1 \%$ & $70.7 \%$ & 0.706 \\
\hline Volume of $\mathrm{ATD}^{>6 \mathrm{~s}}(\mathrm{~mL})$ & 27.77 & $88.2 \%$ & $63.6 \%$ & $75.9 \%$ & 0.777 \\
\hline Volume of ATD ${ }^{2-6 s}(\mathrm{~mL})$ & 38.28 & $82.4 \%$ & $81.8 \%$ & $82.1 \%$ & 0.906 \\
\hline rCBF & 1 & $58.8 \%$ & $90.9 \%$ & $74.9 \%$ & 0.709 \\
\hline $\mathrm{rCBV}^{2-6 s}$ & 1.6 & $88.2 \%$ & $81.8 \%$ & $85.0 \%$ & 0.900 \\
\hline $\mathrm{PCl}^{\mathrm{a}}$ & 61.70 & $88.2 \%$ & $100.0 \%$ & $94.1 \%$ & 0.973 \\
\hline
\end{tabular}

${ }^{a} \mathrm{PCl}=$ Volume of $\mathrm{ATD}^{2-6}$ seconds $\times \mathrm{rCBV}^{2-6}$ seconds

collaterals with a diagnostic accuracy of $94 \%$ in comparison with baseline DSA.

There are several studies on the use of MR perfusion for assessing the collateral status in patients with acute ischemic stroke with predominant focus on the use of perfusion time maps. In 2008, Bang et $\mathrm{al}^{5}$ showed that patients with good collaterals had a larger volume of mild ( 2 seconds $\leq$ Tmax $<4$ seconds) delayed perfusion, but they found no relationship between collateral status and perfusion-diffusion mismatch using Tmax $\geq 4$ seconds. Later, in 2013, Campbell et al ${ }^{12}$ showed that better baseline collateral flow measured by digitally subtracted perfusion MR imaging was associated with a larger diffusion-perfusion mismatch using Tmax $>6$ seconds. In 2014, good collateral status was shown to be associated with a smaller volume of severe hypoperfusion using 


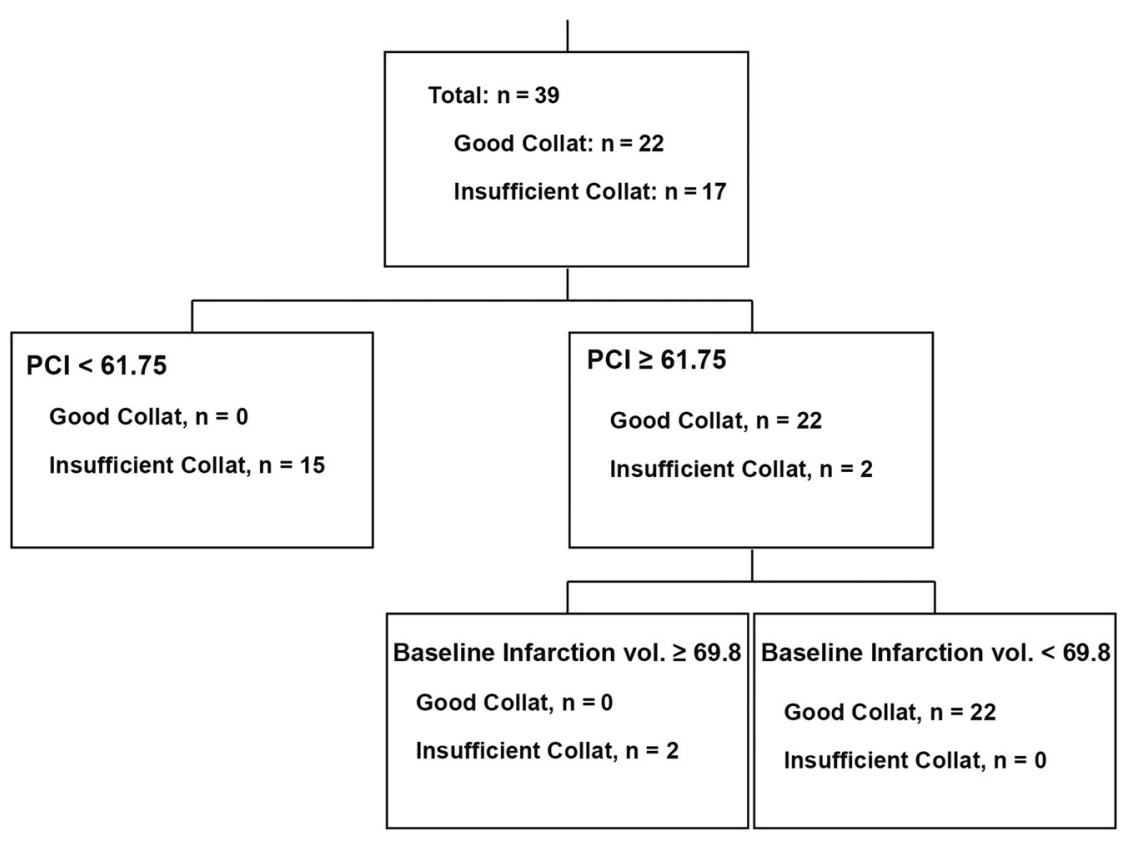

FIG 3. The Classification Tree model using a combination of the PCI (Volume of ATD ${ }^{2-6}$ seconds $X$ $\mathrm{rCBV}^{2-6}$ seconds) and baseline infarction volume provides a nominal (not validated) $100 \%$ accuracy and $\mathrm{ROC}$ area $=1.0$.

Tmax $\geq 6$ seconds ${ }^{13}$ and ATD $\geq 6$ seconds. ${ }^{19}$ Later, Olivot et $\mathrm{al}^{23}$ showed that a smaller hypoperfusion intensity ratio (defined as the proportion of Tmax $>6$-second lesion volume with a Tmax $>10$-second delay) was associated with good collateral status. Most interesting, in 2015, by applying a probabilistic method, Lee et al ${ }^{14}$ showed that good collaterals were associated with more severe delayed perfusion with a Tmax of $16-22$ seconds.

In our study, in addition to commonly used perfusion time maps, we incorporated additional information obtained via rCBV to improve our prediction of collateral status. The hallmarks of collateral perfusion are delay and dispersion. ${ }^{24}$ Perfusion deficits noted on MR perfusion time maps (such as Tmax) encompass both delayed perfusion due to underlying arterial occlusion and delayed flow via collateral circulation. The addition of CBV to routinely used time maps may provide information regarding the amount of blood flow to the ischemic region of the brain (dispersion component); although the arterial source of sustained perfusion may not be evident, in the presence of large arterial occlusion, most of it will be from the collateral circulation.

We showed that in the presence of proximal arterial occlusion, rCBV provides essential information about the status of collaterals and is insightful concerning the distribution and dispersion of collateral flow, a component that may be overlooked using perfusion time maps alone. We showed that mean $\mathrm{rCBV}$ values calculated from the region with moderate hypoperfusion have an overall diagnostic performance (85\%) similar to that of the volume of $\mathrm{ATD}^{2-6}$ seconds $(82.1 \%)$ for predicting good baseline collaterals. However, when used in combination with our newly defined PCI (Volume of $\mathrm{ATD}^{2-6}$ seconds $\times \mathrm{rCBV}^{2-6}$ seconds), it further improved the overall diagnostic performance to $94.1 \%$. This translated into identifying 4 additional (20\%) patients with good collaterals who were falsely categorized by $\mathrm{rCBV}$ or a volume of $\mathrm{ATD}^{2-6}$ seconds alone.

Our results also showed that a larger volume of moderate (ATD ${ }^{2-6}$ seconds $)$ hypoperfusion is a better indicator of good baseline collateral status, with higher specificity $(81.8 \%)$ compared with a smaller volume of severe ( $\mathrm{ATD}^{>6}$ seconds $)$ hypoperfusion (specificity of $63.6 \%$ ). This finding is interesting and somewhat different from prior reports in which a better association between good collateral status and a smaller volume of severe hypoperfusion has been shown using Tmax $>6$ seconds. ${ }^{12,13,23}$ This may be explained by intrinsic technical differences between Bayesian-estimated ATD and singular value decomposition-estimated Tmax.

To increase the broad acceptance of perfusion imaging in the stroke neurology community, further improvement of methodology in image postprocessing is required, especially when dealing with a noisy imaging environment such as DSC perfusion. In this study, we used ATD rather than Tmax to take advantage of inherent noise-resistance behavior of the Bayesian postprocessing. Although Bayesian-derived ATD is equivalent to deconvolution-derived Tmax in terms of definition (maximum time-to-peak of the residue function), they do not share the same properties. The Bayesian method is a robust probabilistic method that minimizes effects of oscillation, tracer delay, and low SNR during residue function estimation compared with other deconvolution methods. ${ }^{18,25}$ The inherent delay insensitivity of the Bayesian technique is essential for accurate evaluation of the perfusion time delay to minimize the effect of existing underlying arterial occlusion. ${ }^{26}$ In addition, simulation studies in agreement with the recommendations from the Acute Stroke Research Imaging Roadmap II ${ }^{27}$ have shown highly reproducible and accurate data for ATD estimation. ${ }^{18} \mathrm{~A}$ follow-up study between ATD and Tmax-estimated PCI may be helpful for exploring potential differences between these techniques.

Our study has several limitations. First, we acknowledge potential selection bias associated with retrospective studies. Second, our small sample size may affect some part of our results. For example, we showed that patients with good baseline collaterals tend to have a higher recanalization rate, but this trend was not statistically significant $(P=.19)$, which may have been a function of the small sample size. In our Classification Tree model, combining PCI and baseline infarct volume yielded a nominal accuracy of 100\% (Fig 3). However, this result must be interpreted in the context of our small sample, and like any predictive model, our results need to be validated in a large prospective cohort. Finally, we excluded 61 patients due to inadequate DSA data for collateral scoring. This is an inevitable challenge in acute ischemic 
stroke because performing a complete diagnostic angiography for collateral assessment poses a delay in treatment. This further highlights the need for development of an advanced noninvasive collateral grading system such as ours from which accurate collateral status may be imputed. To establish the accuracy of our perfusion parameters, we had to compare our results with the current standard of reference (DSA). It is, however, plausible that perfusion imaging may outperform rough collateral grading obtained from 2D DSA as shown by prior reports. ${ }^{12}$

\section{CONCLUSIONS}

Using a multiparametric MR imaging approach, we identified the Perfusion Collateral Index, defined as Volume of ATD ${ }^{2-6 \text { seconds }} \times$ $\mathrm{rCBV}^{2-6}$ seconds, as a new perfusion parameter that provides accurate noninvasive estimation of baseline collateral status with a diagnostic accuracy of $94 \%$ compared with DSA. If its potential is realized, the PCI can be used to accurately identify patients with good collaterals, potentially extending the treatment window and increasing the number of patients who may benefit from endovascular treatment in the current era of endovascular therapy.

Disclosures: Kambiz Nael—UNRELATED: Board Membership: Olea Medical, Comments: Medical Advisory Board; Consultancy: Olea Medical. Thomas NaidichUNRELATED: Consulting Fee or Honorarium: Elsevier. J Mocco-UNRELATED: Consultancy: Rebound Medical, EndoStream, Synchron, Cerebrotech Medical; Other: Apama, The Stroke Project, EndoStream, Synchron, Cerebrotech Medical, Neurvana, NeuroTechnology Investors, Comments: investment. Max Wintermark—UNRELATED: Board Membership: GE-NFL Advisory Board.

\section{REFERENCES}

1. Bang OY, Saver JL, Kim SJ, et al. Collateral flow predicts response to endovascular therapy for acute ischemic stroke. Stroke 2011;42: 693-99 CrossRef Medline

2. Liebeskind DS, Tomsick TA, Foster LD, et al; IMS III Investigators. Collaterals at angiography and outcomes in the Interventional Management of Stroke (IMS) III trial. Stroke 2014;45:759-64 CrossRef Medline

3. Jung S, Gilgen M, Slotboom J, et al. Factors that determine penumbral tissue loss in acute ischaemic stroke. Brain 2013;136:3554-60 CrossRef Medline

4. Galimanis A, Jung S, Mono ML, et al. Endovascular therapy of 623 patients with anterior circulation stroke. Stroke 2012;43:1052-57 CrossRef Medline

5. Bang OY, Saver JL, Buck BH, et al; UCLA Collateral Investigators. Impact of collateral flow on tissue fate in acute ischaemic stroke. J Neurol Neurosurg Psychiatry 2008;79:625-29 Medline

6. Menon BK, O’Brien B, Bivard A, et al. Assessment of leptomeningeal collaterals using dynamic CT angiography in patients with acute ischemic stroke. J Cereb Blood Flow Metabol 2013;33:365-71 CrossRef

7. Lima FO, Furie KL, Silva GS, et al. The pattern of leptomeningeal collaterals on CT angiography is a strong predictor of long-term functional outcome in stroke patients with large vessel intracranial occlusion. Stroke 2010;41:2316-22 CrossRef Medline

8. Hernández-Pérez M, Puig J, Blasco G, et al. Dynamic magnetic resonance angiography provides collateral circulation and hemodynamic information in acute ischemic stroke. Stroke 2016;47:531-34 CrossRef Medline

9. Ernst M, Forkert ND, Brehmer L, et al. Prediction of infarction and reperfusion in stroke by flow- and volume-weighted collateral signal in MR angiography. AJNR Am J Neuroradiol 2015;36:275-82 CrossRef Medline
10. Shuaib A, Butcher K, Mohammad AA, et al. Collateral blood vessels in acute ischaemic stroke: a potential therapeutic target. Lancet Neurol 2011;10:909-21 CrossRef Medline

11. Wintermark M, Rowley HA, Lev MH. Acute stroke triage to intravenous thrombolysis and other therapies with advanced CT or MR imaging: pro CT. Radiology 2009;251:619-26 CrossRef Medline

12. Campbell BC, Christensen S, Tress BM, et al; EPITHET Investigators. Failure of collateral blood flow is associated with infarct growth in ischemic stroke. J Cereb Blood Flow Metabol 2013;33:1168-72 CrossRef Medline

13. Marks MP, Lansberg MG, Mlynash M, et al; Diffusion and Perfusion Imaging Evaluation for Understanding Stroke Evolution 2 Investigators. Effect of collateral blood flow on patients undergoing endovascular therapy for acute ischemic stroke. Stroke 2014;45:1035-39 CrossRef Medline

14. Lee MJ, Son JP, Kim SJ, et al. Predicting collateral status with magnetic resonance perfusion parameters: probabilistic approach with a Tmax-derived prediction model. Stroke 2015;46: 2800-07 CrossRef Medline

15. Keedy AW, Fischette WS, Soares BP, et al. Contrast delay on perfusion CT as a predictor of new, incident infarct: a retrospective cohort study. Stroke 2012;43:1295-301 CrossRef Medline

16. Vagal A, Menon BK, Foster LD, et al. Association between CT angiogram collaterals and CT perfusion in the Interventional Management of Stroke III Trial. Stroke 2016;47:535-38 CrossRef Medline

17. Kim SJ, Son JP, Ryoo S, et al. A novel magnetic resonance imaging approach to collateral flow imaging in ischemic stroke. Ann Neurol 2014;76:356-69 CrossRef Medline

18. Boutelier T, Kudo K, Pautot F, et al. Bayesian hemodynamic parameter estimation by bolus tracking perfusion weighted imaging. IEEE Trans Med Imaging 2012;31:1381-95 CrossRef Medline

19. Nicoli F, Scalzo F, Saver JL, et al; UCLA Stroke Investigators. The combination of baseline magnetic resonance perfusion-weighted imagingderived tissue volume with severely prolonged arterial-tissue delay and diffusion-weighted imaging lesion volume is predictive of MCA-M1 recanalization in patients treated with endovascular thrombectomy. Neuroradiology 2014;56:117-27 CrossRef Medline

20. Dardzinski BJ, Sotak CH, Fisher M, et al. Apparent diffusion coefficient mapping of experimental focal cerebral ischemia using diffusion-weighted echo-planar imaging. Magn Reson Med 1993;30: 318-25 CrossRef Medline

21. Higashida RT, Furlan AJ, Roberts H, et al; Technology Assessment Committee of the American Society of Interventional and Therapeutic Neuroradiology, Technology Assessment Committee of the Society of Interventional Radiology. Trial design and reporting standards for intra-arterial cerebral thrombolysis for acute ischemic stroke. Stroke 2003;34:e109-37 CrossRef Medline

22. Tomsick T, Broderick J, Carrozella J, et al; Interventional Management of Stroke II Investigators. Revascularization results in the Interventional Management of Stroke II trial. AJNR Am J Neuroradiol 2008;29:582-87 CrossRef Medline

23. Olivot JM, Mlynash M, Inoue $\mathbf{M}$, et al; DEFUSE 2 Investigators. $\mathbf{H y -}$ poperfusion intensity ratio predicts infarct progression and functional outcome in the DEFUSE 2 cohort. Stroke 2014;45:1018-23 CrossRef Medline

24. Liebeskind DS. Collateral circulation. Stroke 2003;34:2279-84 CrossRef Medline

25. Mouridsen K, Friston K, Hjort N, et al. Bayesian estimation of cerebral perfusion using a physiological model of microvasculature. Neuroimage 2006;33:570-79 CrossRef Medline

26. Sasaki M, Kudo K, Boutelier T, et al. Assessment of the accuracy of a Bayesian estimation algorithm for perfusion CT by using a digital phantom. Neuroradiology 2013;55:1197-203 CrossRef Medline

27. Wintermark M, Albers GW, Broderick JP, et al. Acute Stroke Imaging Research Roadmap II. Stroke 2013;44:2628-39 CrossRef Medline 